\title{
From left atrial pressures to left atrial strain: The importance of diastolic dysfunction in patients with atrial fibrillation
}

\author{
Luca Longobardo ${ }^{1,2}$, Concetta Zito ${ }^{1}$, Scipione Carerj ${ }^{1}$ \\ 'Section of Cardiology, Department of Clinical and Experimental Medicine, University of Messina, Azienda Ospedaliera Universitaria 'Policlinico G. Martino' and Uni- \\ versità degli Studi di Messina, Messina, Italy \\ ${ }^{2}$ Section of Cardiology, Presidio Ospedaliero Piemonte, IRCCS Bonino-Puejo, Viale Europa, Messina, Italy
}

\section{Related article}

by Uziębło-Życzkowska et al.

see p. 1223

\author{
Correspondence to: \\ Luca Longobardo, MD, PhD, \\ Section of Cardiology, \\ Department of Clinical and \\ Experimental Medicine, \\ Policlinico G. Martino, Via \\ Consolare Valeria n 12, 98100 \\ Messina, Italy, \\ phone: +39 902212969 \\ e-mail: \\ lucalongobardo87@gmail.com \\ Copyright by the Author(s), 2021 \\ Kardiol Pol. 2021; \\ 79 (11): 1195-1196; \\ DOI: 10.33963/KP.a2021.0145 \\ Received: \\ October 15, 2021 \\ Revision accepted: \\ October 15, 2021 \\ Published online: \\ November 2, 2021
}

In 2021 atrial fibrillation (AF) is the most common sustained cardiac arrhythmia in adults which affects between $2 \%$ and $4 \%$ of the adult population [1]. The onset of atrial fibrillation is closely related to left atrial changes, including the substitution of myocardial cells with collagen fibers and atrial enlargement, which allow the creation of re-entrant circuits, the fundamental background for this arrhythmia. The main trigger for atrial remodeling is the increase of left ventricular (LV) filling pressures. Pathological conditions like hypertension, diabetes, and obesity, or the physiological progressive aging, make the left ventricle stiff and less compliant, with a consequent increase of LV filling pressures that has earlier repercussions on the left atrium $(L A)$, less resistant to high pressures than the LV. As a consequence, the LA starts to enlarge, substituting muscle cells with collagen fibers while becoming stiffer at the same time [2]. In this context, it is quite evident that the assessment of left atrial pressure (LAP) becomes of great importance for the clinical management of these patients. Nowadays, there are two methods for assessing LAP. The first one is the percutaneous cardiac catheterization, which allows an accurate evaluation of right and left side intracardiac pressures but is an invasive technique that is currently reserved only for well-determined cases; the second one is the indirect estimation of LAP by echocardiography. According to the current international guidelines [3], the estimation of diastolic function, of which LAP is one of the major determinants, by echocardiography can be performed using a step-by-step algorithm, including the measurements of a ratio of mitral peak early (E) to late (A) velocity; septal and lateral early diastolic mitral annular velocities $\left(\mathrm{e}^{\prime}\right)$ and the ratio of $\mathrm{E}$ to the averaged value of $\mathrm{e}^{\prime}\left(\mathrm{E} / \mathrm{e}^{\prime}\right)$; the maximum tricuspid regurgitation (TR) velocity and the left atrial volume index (LAVi). On the basis of these elements, diastolic dysfunction can be classified into grades I, II, and III, with or without increased LAP.

Unfortunately, in real life, things are not so easy as each of these parameters suffers from significant limitations. LAVi provides only anatomical information and is rarely increased in acute diseases like myocardial infarction [4]; $E / E$ ' ratio is characterized by a too large "grey zone", which includes a significant percentage of patients; TR peak velocity is often undetectable because of the absence of tricuspid regurgitation or low-quality images. Therefore, although these weaknesses are compensated by the proposed step-by-step algorithm, finding discordant results within the suggested parameters is common and frequently leads to the inability to determine the degree of LV diastolic dysfunction and LAP. In this context, the assessment of LA function by 2D speckle tracking echocardiography (STE) showed to have a significant role in a more accurate evaluation of diastolic function. Indeed, Singh et al. showed that the use of well-defined LA strain thresholds significantly improved the assessment of diastolic dysfunction grades in unselected patients with preserved LV ejec- 
tion fraction [5]. Within the parameters that left atrial 2D STE analysis provides, the LA reservoir is the most accurate one as it depends mostly on LA relaxation due to both the LV filling pressure and the amount of fibrosis.

In accordance with the above, in their recent article, Uziębło-Życzkowska et al. [6] found a close correlation between LAP, measured during atrial fibrillation ablation, and LA reservoir strain. The authors enrolled 172 patients with atrial fibrillation undergoing ablation and divided them according to the LA strain reservoir value into three terciles; then they compared patients with lower values (first tercile) with those with higher values (third tercile) and found that the former were older and more often women, they had a larger LA and showed a more impaired LV diastolic function. These data are of great interest. First of all, the authors demonstrated that 2D STE left atrial strain is a feasible tool for the assessment of LAP and thus a reliable parameter for the evaluation of LV diastolic function. Secondly, they described the main features of patients that showed a worse diastolic dysfunction - the same patients that have an increased risk of recurrence after ablation and worse prognosis, as already described in the literature [7]. These data, if confirmed in larger studies, could be the basis for the creation of risk scores, including clinical and echocardiographic parameters, for the prediction of prognosis after atrial fibrillation ablation.

At a time when atrial fibrillation is the most common arrhythmia in adults, a more accurate assessment of diastolic dysfunction using parameters like 2D STE left atrial strain, together with the evaluation of clinical risk factors, could be a strong weapon in the armory of a cardiologist for a correct and more and more tailored management of these patients.

\section{Article information}

Conflict of interest: None declared.

Open access: This article is available in open access under Creative Common Attribution-Non-Commercial-No Derivatives 4.0 International (CC BY-NC-ND 4.0) license, allowing to download articles and share them with others as long as they credit the authors and the publisher, but without permission to change them in any way or use them commercially. For commercial use, please contact the journal office at kardiologiapolska@ptkardio.pl.

How to cite: Longobardo L, Zito C, Carerj S. From left atrial pressures to left atrial strain:The importance of diastolic dysfunction in patients with atrial fibrillation. Kardiol Pol. 2021; 79(11): 1195-1196, doi: 10.33963/KP.a2021.0145.

\section{REFERENCES}

1. Benjamin EJ, Muntner $P$, Alonso A, et al. Heart Disease and Stroke Statistics-2019 Update: A Report From the American Heart Association. Circulation. 2019; 139(10): e56-e5e528, doi: 10.1161/CIR.0000000000000659, indexed in Pubmed: 30700139.

2. Longobardo L, Zito C, Carerj S, et al. Left atrium in heart failure with preserved ejection fraction: the importance of function before anatomy. Eur Heart J Cardiovasc Imaging. 2017; 18(7): 730-731, doi: 10.1093/ehjci/jex059, indexed in Pubmed: 28379463.

3. Nagueh SF, Smiseth $\mathrm{OA}$, Appleton $\mathrm{CP}$, et al. Recommendations for the Evaluation of Left Ventricular Diastolic Function by Echocardiography: An Update from the American Society of Echocardiography and the European Association of Cardiovascular Imaging. J Am Soc Echocardiogr. 2016; 29(4): 277-314, doi: 10.1016/j.echo.2016.01.011, indexed in Pubmed: 27037982.

4. Longobardo L, Zito C, Khandheria BK. Left atrial function index: did we end up waiting for Godot? Eur Heart J Cardiovasc Imaging. 2017; 18(2): 128-129, doi: 10.1093/ehjci/jew210, indexed in Pubmed: 28115410.

5. Singh A, Addetia K, Maffessanti F, et al. LA strain for categorization of LV diastolic dysfunction. JACC Cardiovasc Imaging. 2017; 10(7): 735-743, doi: 10.1016/j.jcmg.2016.08.014, indexed in Pubmed: 28017389.

6. Uziębło-Życzkowska B, Krzesiński P, Jurek A, et al. Correlations between left atrial strain and left atrial pressures values in patients undergoing atrial fibrillation ablation. Kardiol Pol. 2021; 79(11): 1223-1230, doi: 10.33963/KP.a2021.0113, indexed in Pubmed: 34599496.

7. Motoki $H$, Negishi $K$, Kusunose $K$, et al. Global left atrial strain in the prediction of sinus rhythm maintenance after catheter ablation for atrial fibrillation. J Am Soc Echocardiogr. 2014; 27(11): 1184-1192, doi: 10.1016/j. echo.2014.08.017, indexed in Pubmed: 25260436. 